\title{
Epidermal Cysts Surgery: Case Series
}

\author{
Teodor Angelov ${ }^{*}$, Valeri Malev², Georgi Tchernev² \\ ${ }^{1}$ Student, state trainee, Faculty of Medicine, Medical University of Sofia, Bulgaria \\ ${ }^{2}$ Onkoderma- Clinic for Dermatology, Venereology and Dermatologic Surgery, General Skobelev, Sofia, Bulgaria
}

Received: August 17, 2020; Accepted: August 19, 2020; Published: August 21, 2020

*Corresponding author: Professor Georgi Tchernev, PhD, Onkoderma-Clinic for Dermatology, Venereology and Dermatologic Surgery, Sofia, Bulgaria, Tel. No: 00359885588424; E-mail: georgi_tchernev@yahoo.de

\begin{abstract}
Epidermal cysts are the most common scalp cysts in clinical practice. They are most commonly located in the region of the face, neck, and trunk, and have been reported in many other topographic areas of the body. Clinically, they are present as free-moving nodules located below the skin, ranging in size from a few millimeters to a few centimeters in diameter. Histopathologically, they are defined as benign tumor formations with a very low risk of malignancy. The differential diagnosis includes: lipoma, neurofibroma, salivary gland adenoma, vascular lesion and fibroid, and Trichilemmal cyst. The most common treatment for epidermal cysts is surgical excision with removal of all contents without compromising the integrity of the capsule. In this publication, we present five clinical cases of patients with epidermal cysts in different topographic regions of the body. The patients were treated surgically by elliptical excision and complete removal of epidermal cysts without rupture. The defects were closed with single discontinued skin sutures and the sutures were removed on an 8- / 14-postoperative day. No recurrence was observed in the postoperative period.
\end{abstract}

\section{Introduction}

Epidermal cysts, also known as epidermoid, infundibular and keratin, are the most common skin cysts [1]. They can be form all over the body, the most common localizations being the face, neck and trunk [2]. They occur in the infundibulum of the hair follicle. They present clinically as nodules just below the skin with visible central puncture. Their size varies from a few millimeters to a few centimeters in diameter and are freely movable [1].

We present five clinical cases of patients with epidermal cysts in different topographic regions of the body - in the back area (regio lumbalis and regio scapularis), in the head area(regio occipitalis), in the ear area (regio auricularis) and in the area of the inguinal canal (regio inguinalis sinistra).

\section{Description of the Clinical Cases}

The first patient (Figure 1a-1c, Figure 2a-2c) has two atheromas on the back, bilaterally (left and right), in the area of the scapula (regio scapularis). The patient is 44 years old, with no evidence of comorbidities, allergies or medications. Anamnestic reports of itching, discomfort and slight redness in both scapular areas. Clinical and ultrasound data correspond to a benign tumor formation located on the muscles - atheroma. The formation is removed surgically, under local anesthesia with Lidocaine, with excision to the muscles, cessation of bleeding and stretching plasticity. The defect is closed with single skin sutures. The sutures are removed on the 14th postoperative day, without complications and without evidence of recurrence.

The second patient (Figure 3a-3b) has an atheroma on the back, in the area of the scapula (regio scapularis). The patient is 60 years old, with no evidence of comorbidities or allergies. The patient is taking antihypertensive therapy - Lercanidipine $10 \mathrm{mg}$ (1-0-1) and Valsartan / Hydrochlorothiazide 160mg / 12.5mg (1$0-0$ ) since August 2013. Two years later, he underwent surgery for a keratinocyte tumor in the back, histologically verified as basal cell carcinoma. The patient is admitted for surgical treatment of two lesions - epidermal cyst and dysplastic nevus. On clinical examination, the epidermal cyst is localized in the shoulder area, $5 \mathrm{~cm}$ in diameter, soft, rounded, red, slightly mobile. The formation was removed surgically, under local anesthesia with Lidocaine, with deep elliptical excision and excision to the muscles, hemostasis and stretching plasticity. The defect is closed with single continuous skin sutures. Histopathological examination shows a ruptured epidermal cyst. The sutures are removed on the 14th postoperative day, without complications and without evidence of recurrence.

The third patient (Figure 4a-4e) has an epidermal cyst in the head area (regio occipitalis). The patient is 43 years old. According to anamnestic data, the cyst has appeared 4 years ago and has been growing steadily ever since. Several times the cyst becomes inflamed, which is expressed in pain, redness and bright, semi-liquid secretion. As concomitant diseases, the patient reports vitiligo on the hands, dating back to his childhood. 

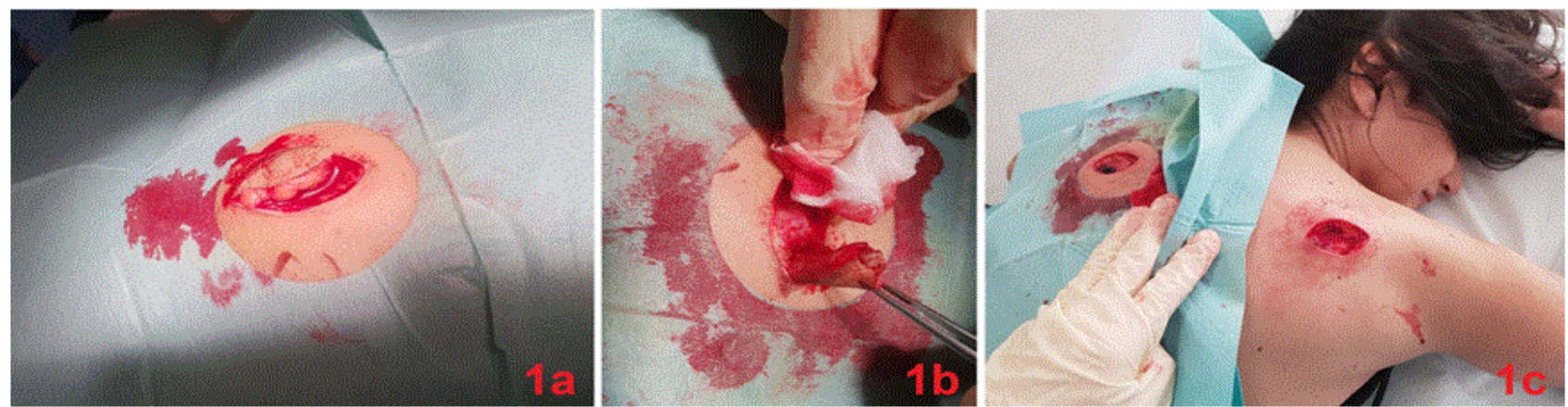

Figure 1a-c: Intraoperative view of a resected epidermal cyst in the scapular region (regio scapularis) of a 44-year-old patient.
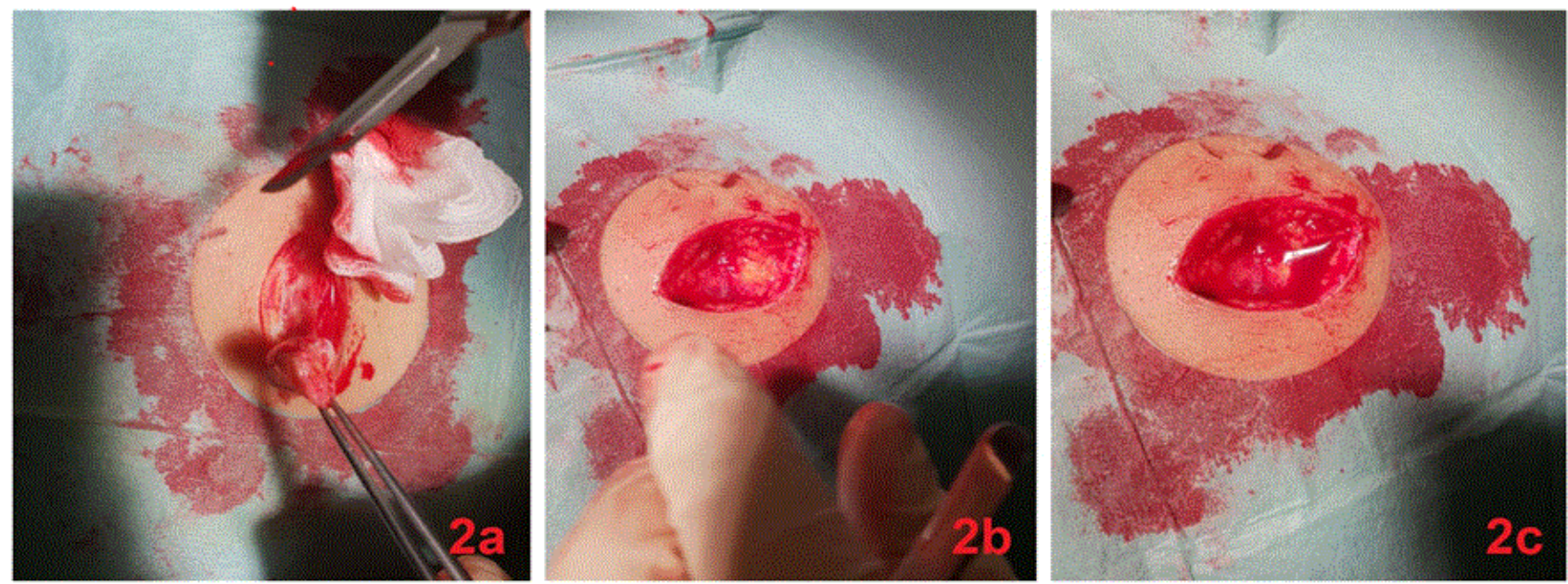

Figure 2a-c: Intraoperative view of a resected epidermal cyst in the scapular region (regio scapularis) of a 44-year-old patient.
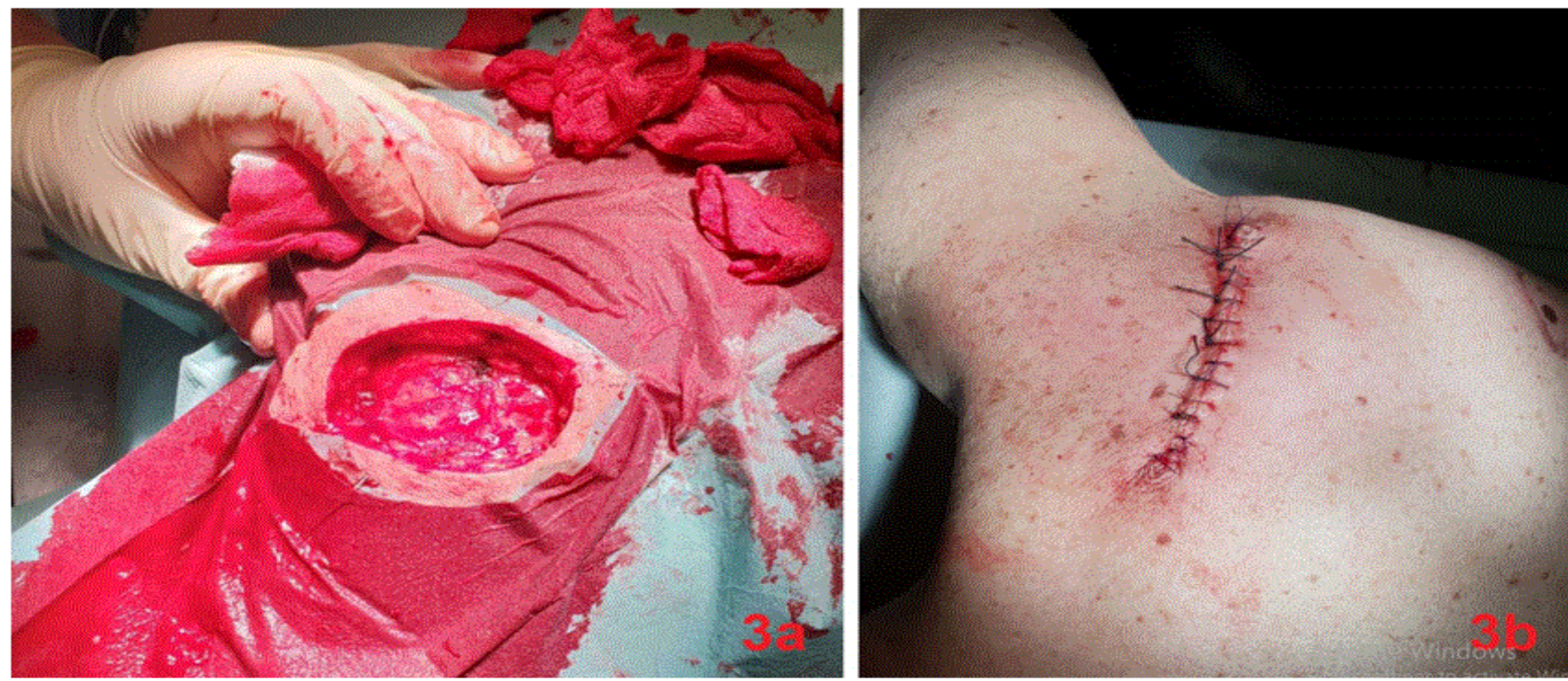

Figure 3a-b: Intraoperative view of a resected epidermal cyst in the scapular area (regio scapularis) of a 60-year-old patient. b: Postoperative view of a closed operative wound with single skin sutures. 

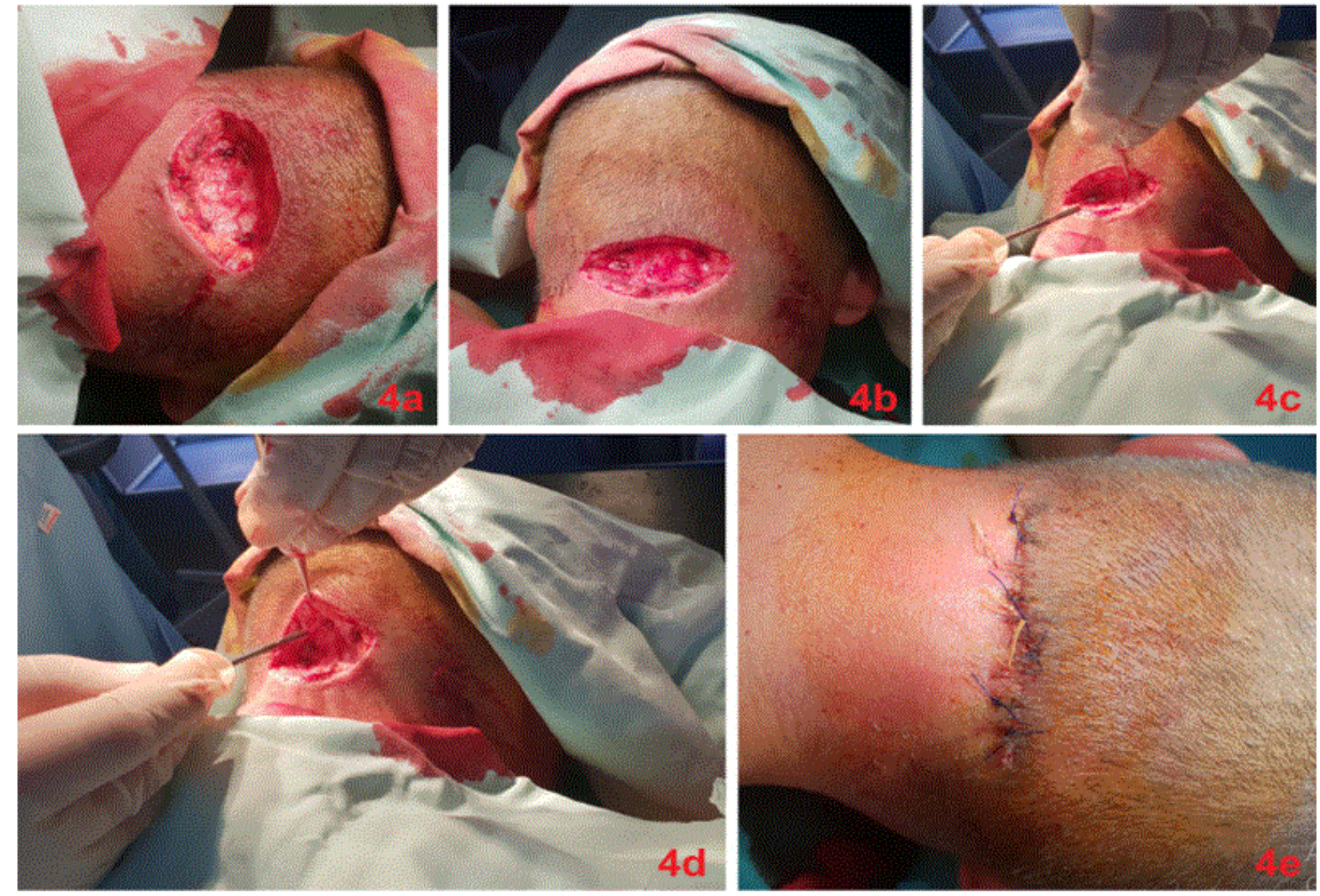

Figure 4a-e: Intraoperative view of a resected epidermal cyst in the head area (regio occipitalis) of a 43-year-old patient. 4e: postoperative view closed operative wound with a classic, interrupted suture

In the clinical examination, the cyst is $5 \mathrm{~cm}$ in diameter, soft, slightly movable, located on the border between the occipital bone and the sternocleidomastoid muscle. In addition, during the examination the formation is in a stage of inflammation.The cyst is surgically removed by elliptical excision under local anesthesia with lidocaine. There is no data on postoperative neurological complications and limitations in head mobility. The sutures were removed on the 14th postoperative day, without complications and without evidence of recurrence.

The fourth patient (Figure 5a-5d) has an epidermal cyst in the ear area (regio auricularis). The patient is 46 years old, and from the objective condition there is evidence of a mild form of adipositas per magna. Anamnestic, the patient reports pain near the lower part of the auricle. There is no data on concomitant diseases, concomitant medication and allergies. Clinical examination and imaging (ultrasound) correspond to the diagnosis of epidermal cyst. Paraclinical examinations are normal. The epidermal cyst is surgically removed under local anesthesia after its preparation. The defect is closed with single skin sutures. The sutures are removed on the 8th postoperative day, without complications. There is a perfect healing of the defect, no recurrence.

The fifth patient (Figure 6a-6d, Figure 7a-7d) has an epidermal cyst in the area of the inguinal canal (regio inguinalis sinistra). The patient is 70 years old, with Valsartan-induced stage IV melanoma, currently with metastases to the liver and draining lymph nodes, with a regressive course and therapy with BRAFand MEK-inhibitors. The general condition of the patient is stable. Anamnestic evidence of painful redness in the lower abdomen, erythema, edema and burning. Clinical examination and imaging (ultrasound examination) correspond to the diagnosis of epidermal cyst or atheroma, as the formation is unruptured and located above the muscles. Paraclinical examinations are without pathological changes. The cyst is surgically dissected and the defect is closed with single skin sutures. The sutures were removed on the 14 th postoperative day, without complications and without evidence of recurrence. 

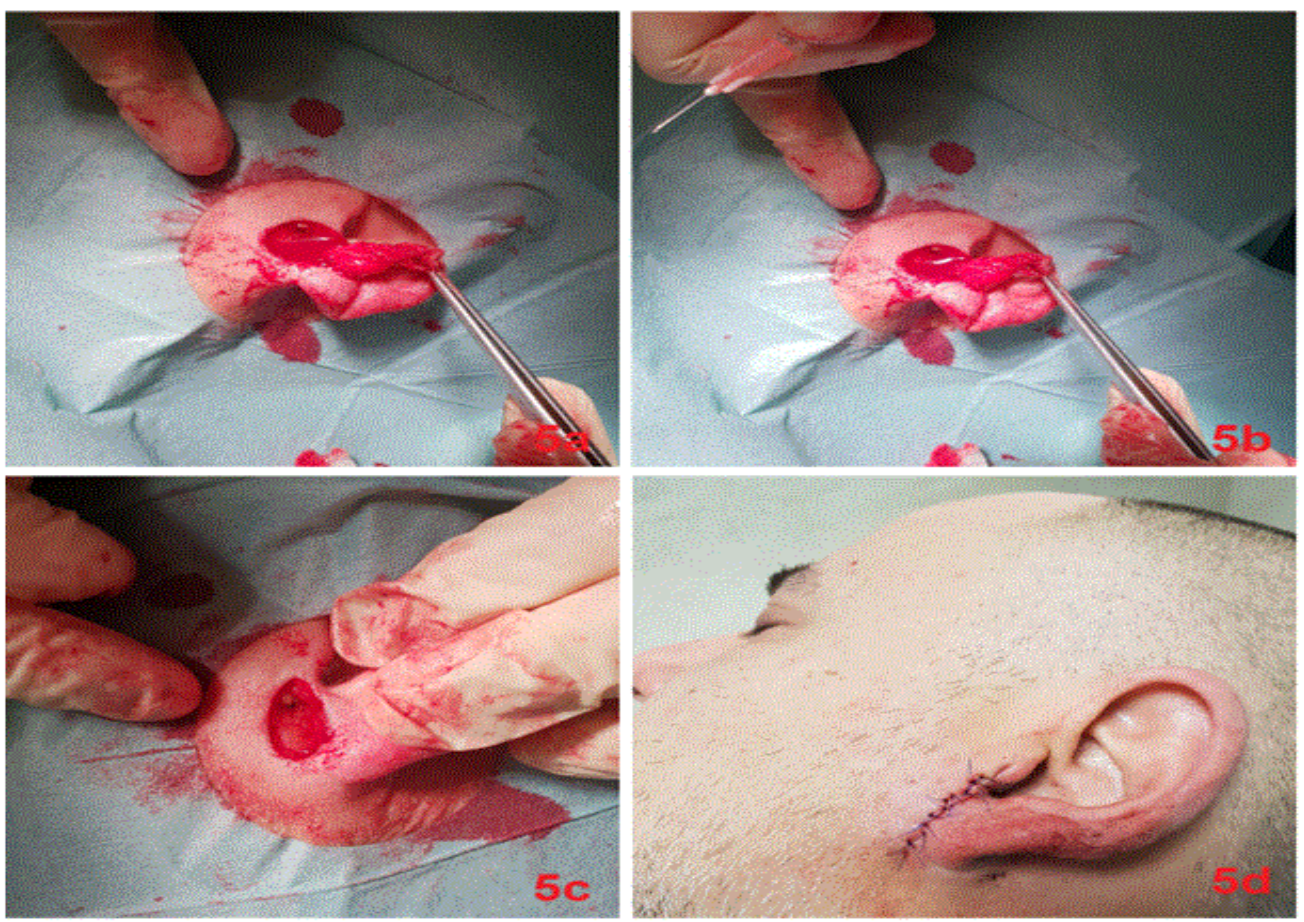

Figure 5a-c: Intraoperative view of a resected epidermal cyst in the ear area (regio auricularis) of a 46-year-old patient. d: Postoperative view of a closed operative wound with single skin sutures.
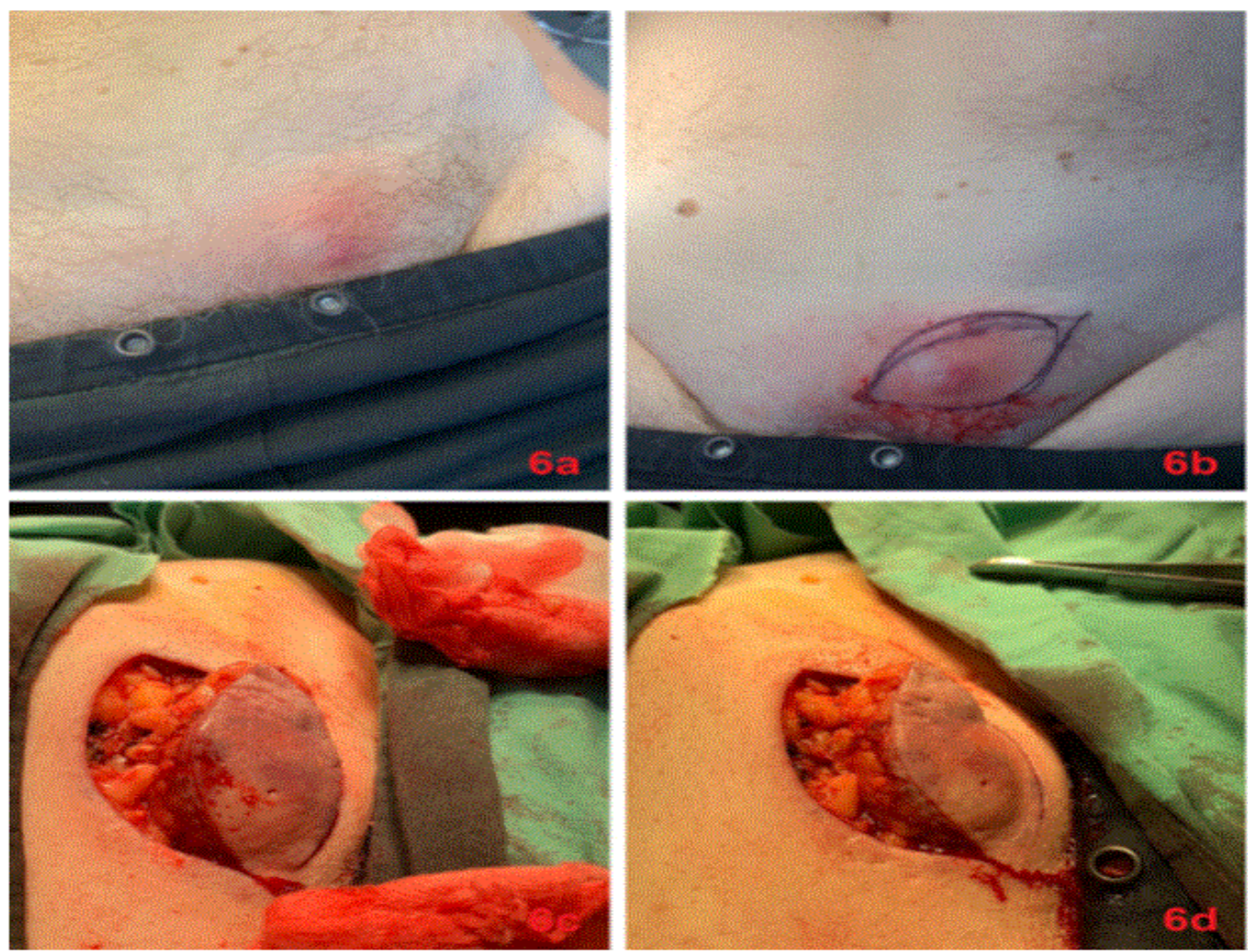

Figure 6a- b: Preoperative view of an epidermal cyst in the area of the inguinal canal (regio inguinalis sinistra) of a 70-year-old patient. Figure 6c- d: Intraoperative view of the resected epidermal cyst. 

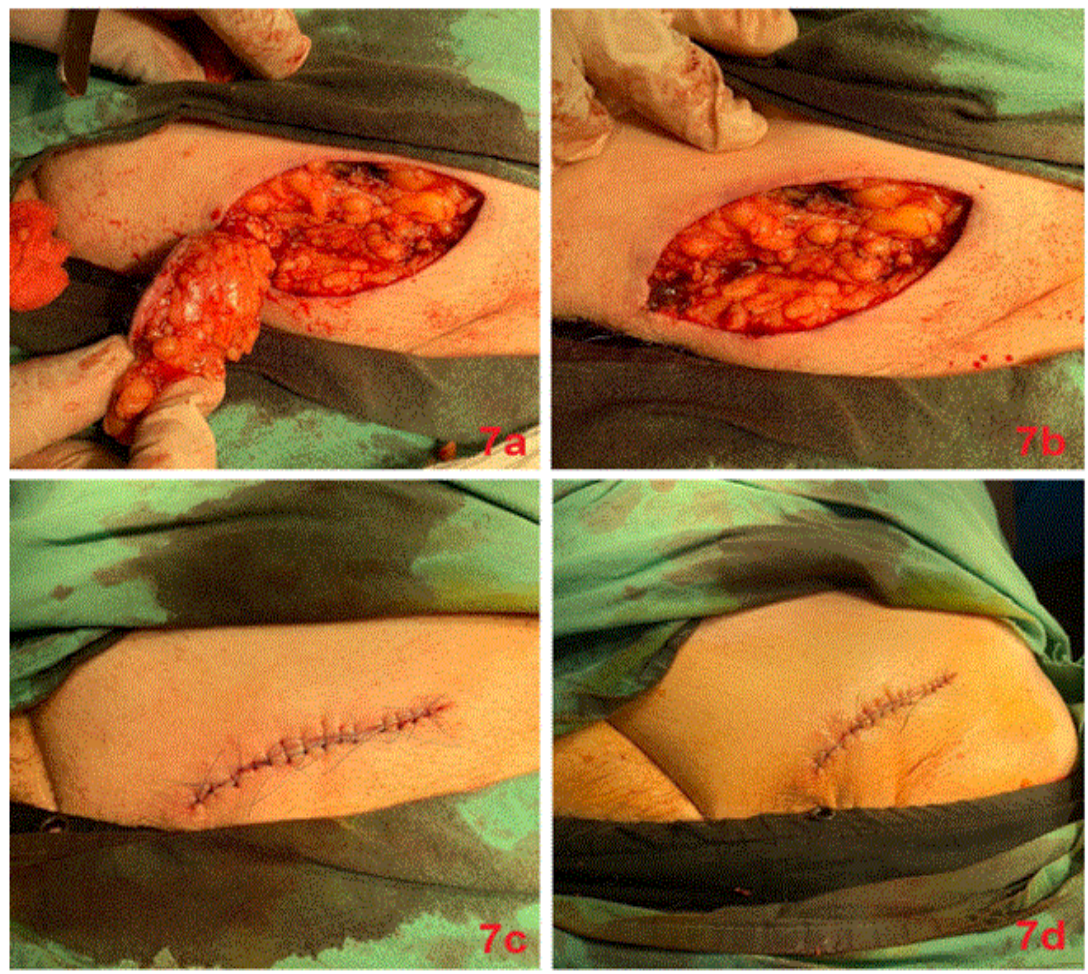

Figure 7a- b: Intraoperative view of a resected epidermal cyst in the area of the inguinal canal (regio inguinalis sinistra) of a 70-year-old patient. Figure 7c- d: Postoperative view of a closed operative wound with single skin sutures.

\section{Discussion}

Epidermal cysts are congenital and acquired [3]. Congenital cysts are usually located along the midline of the body and arise from the proliferation of closed ectodermal cells during early embryogenesis [3]. Acquired cysts are located mainly in the head and neck [4]. They are defined as benign formations with a very low risk of malignancy, as a study by Bauer et al. shows that $2.2 \%$ of them can develop into well-differentiated squamous cell carcinoma $[4,5]$. Therefore, all epidermal cysts should be evaluated histopathologically after surgical excision [5].

The pathogenetic mechanism of acquired epidermal cysts involves the implantation of epidermal cells into deep mesenchymal tissues, induced by various factors such as local trauma, constant pressure on the skin, local inflammation of the hair follicle, and even insect bites [6]. In the presented patients the most probable factors for the formation of an epidermal cyst are the prolonged pressure and the inflammation between the skin folds in the separate topographic areas. Histologically, at the center of the cyst, there is an accumulation of keratin rather than sebum, despite the misuse of the term sebaceous cyst because the cysts do not originate from the sebaceous glands $[7,8]$. Macroscopically, the accumulation of keratin has a "cheesy" appearance [8]. Their progression may proceed at different rates over time over years or be stationary [6]. In most cases, their wall is thin and when it bursts, complications such as inflammation and stiffness can develop. When inflamed, they increase in size and color and are more likely to be recognized by the patient as painful, subcutaneous nodules.There are no scientifically proven predictive factors as to whether they will expand, become inflamed or remain steady [1].

Histopathologically epidermal cysts should also be distinguished from trichilemal cysts. Trichilemmal cysts are surrounded by fibrous capsules covered with small, cuboidal, dark-colored, palisade-arranged basal epithelial cells, with no distinct cell bridges between them [9].

They fuse with characteristic squamous epithelium composed of swollen pale keratinocytes, gradually increasing in height and abruptly transforming into solid eosinophilic keratin, often with foci of calcification without forming a granular cell layer. In contrast, epidermoid cysts contain a granular cell layer. The nonimplantation variant of epidermoid cysts contains laminates of keratin, which are thought to be formed by the infundibulum of the hair follicle [9].

Although their most common localizations are in the face area, neck, and trunk, there are scientifically reported cases of epidermal cysts in the scrotum, genitals, fingers, and buccal mucosa [4]. Infection of the ducts of the eccrine glands with HPV can cause the formation of cysts on the palms and feet, and many cysts on the body may be associated with Gardner's syndrome [6]. The clinical diagnosis can be supported by additional studies such as ultrasonography, fine-needle aspiration biopsy, CT and MRI 
[7]. The differential diagnosis includes: lipoma, neurofibroma, salivary gland adenoma, vascular lesion, and fibroma [10].

The most common treatment for epidermal cysts in clinical practice is surgical excision with removal of all contents, which reduces the risk of recurrence [1]. It is recommended that the excision of the cyst be removed in its entirety to protect the operative field from contamination with its contents. Classical elliptical excision is suitable for most localizations and provides a tension-free operative wound with good cosmetic results [1].

A clinical study conducted by Mehrabi et al. among 646 patients with epidermal and pilar cysts regarding the effectiveness and therapeutic recovery after this type of surgery, confirms the effectiveness, rapid healing of the wound defect and good postoperative cosmetic results [11]. Recurrence varies between 3.6 and $8.3 \%$, with recurrences most common in epidermal cysts in the back area (13.8\%) and in the ear area (13\%) [11].

Lasers, through which fenestration and decompression of the cyst contents are achieved, are also a therapeutic option for epidermal cysts [12]. A study by Kim et al. among 120 patients treated with CO2-laser showes an advantage over surgical excision, both in terms of postoperative scar $(0.30 \pm 0.15 \mathrm{~cm}$ compared to $1.23 \pm 0.43 \mathrm{~cm}$ in surgical technique ( $p<0.001)$ ) and for the technical time of the procedure $(16.15 \pm 5.96$ minutes vs. $22.38 \pm 6.05$ minutes when treated with excision $(p<0.001)$ ) [13]. The minimally invasiveness and better visible postoperative results of $\mathrm{CO} 2$ lasers have been confirmed by many other authors $[14,15]$.

\section{Conclusion}

In conclusion, we inform about 5 cases of cysts from dermatological practice, successfully treated by elliptical excision under local anesthesia, without recurrence within 3 to 5 years of clinical follow-up. Unlike other surgical methods described in the world literature, excisions were performed using the so-called wide ellipse, which allows better dissection of the epidermal cyst in all directions and minimizes the possibility of rupture of the capsule and subsequent recurrences in near or far periods.

\section{Reference}

1. Weir CB and Hilaire NJ. Epidermal Inclusion Cyst. InStatPearls. 2020

2. Zito P, Scharf R. Cyst, Epidermoid (Sebaceous Cyst). StatPearls. 2020.

3. Kandogan T, Koc M, Vardar E, Selek E and Sezgin O. Sublingual epidermoid cyst: a case report. J Med Case Reports. 2007;1:87. doi: 10.1186/1752-1947-1-87.

4. Rao A, Rao B, Kurian MJ and Pai RR. Two rare presentations of epidermal cyst. J Clin Diagn Res. 2014;8(10):OD01-3. doi: 10.7860/ JCDR/2014/8070.4982.

5. Bauer BS and Lewis JV. Carcinoma arising in sebaceous and epidermoid cysts. Ann Plast Surg. 1980;5(3):222-226. doi: 10.1097/00000637198009000-00008.

6. Pandya KA and Radke F. Benign skin lesions: lipomas, epidermal inclusion cysts, muscle and nerve biopsies. Surg Clin North Am. 2009;89(3):677-687. doi: 10.1016/j.suc.2009.03.002

7. Mahalakshmi S, Reddy S, Ramamurthy TK and Shilpa B. Rare locations of epidermoid cyst: case reports and review. Ethiop J Health Sci. 2016;26(6): 595-601. doi: 10.4314/ejhs.v26i6.14

8. Weedon D. Weedon's skin pathology. Elsevier. 3rd ed. London: Elsevier 2010.

9. Kirkham N. Tumors and Cysts of the Epidermis. Lever's Histopathology of the Skin. 10th ed. Philadelphia 2009.801-803.

10. Chen B, Lu H, Ren C, Ma L, Hu X, Qi H, et al. Excision of sebaceous cyst by intraoral approach: A case report. Medicine (Baltimore). 2017;96(49):e8803. doi: 10.1097/MD.0000000000008803.

11. Mehrabi D, Leonhardt JM and Brodell RT. Removal of keratinous and pilar cysts with the punch incision technique: analysis of surgical outcomes. Dermatol surg. 2002 ;28(8):673-677. doi: 10.1046/j.15244725.2002.02020.x.

12. Feng C, Ma H. Treatment of epidermal cysts with erbium: YAG laser fenestration: an alternative to surgical intervention. Ann Plast Surg. 2015;74 Suppl 2:S89-92. doi: 10.1097/SAP.0000000000000463.

13. Kim K, Sun H, Chung E. Comparison of complete surgical excision and minimally invasive excision using $\mathrm{CO} 2$ laser for removal of epidermal cysts on the face. Arch Craniofac Surg. 2019;20(2):84-88. doi: 10.7181/acfs.2018.02152.

14. Alami M, Ghufoor K, Dilkes M. Laser marsipulization of epidermal cysts: avoiding linear scars. J Clin Laser Med Surg. 2003;21(3):161-163. doi: 10.1089/104454703321895626.

15. Song S, Burm J, Yang W, Kang S. Minimally Invasive Excision of Epidermal Cysts through a Small Hole Made by a CO2 Laser. Arch Plast Surg. 2014;41(1):85-88. doi: 10.5999/aps.2014.41.1.85. 